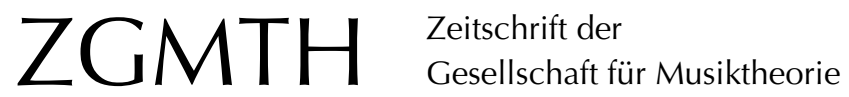

Utz, Christian (2015): Nicholas Cook, Beyond the Score: Music as Performance, New York: Oxford University Press 2013. ZGMTH 12/2, 275-285.

https://doi.org/10.31751/826

(C) 2015 Christian Utz

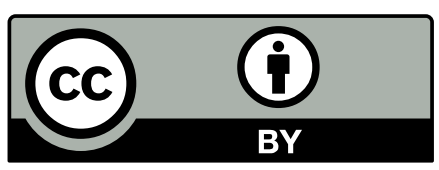

Dieser Text erscheint im Open Access und ist lizenziert unter einer Creative Commons Namensnennung 4.0 International Lizenz.

This is an open access article licensed under a

Creative Commons Attribution 4.0 International License.

eingereicht / submitted: 13/03/2016

angenommen / accepted: 17/03/2016

veröffentlicht / first published: 30/07/2016

zuletzt geändert / last updated: 28/03/2018 


\section{Nicholas Cook, Beyond the Score: Music as Performance, New York: Oxford University Press 2013}

Nicholas Cook hat mit seiner jüngsten Monographie eine eindrucksvolle Serie von Buchpublikationen fortgesetzt, die allesamt für sich beanspruchen können, Musikwissenschaft und Musiktheorie wesentliche Impulse verliehen oder zumindest kontroverse und damit fruchtbare Methodendiskussionen befördert zu haben. ${ }^{1}$ In vielen Texten Cooks ist deutlich die Stoßrichtung hin zu einer Reform, wenn nicht vollständigen Ablösung etablierter methodischer Verfahren vernehmbar, seien sie nun der musikalischen Analyse im engeren Sinn oder etwa der Musikhistoriographie oder Theoriegeschichtsschreibung zuzuordnen. Der reformatorische Impuls verbindet Cooks Arbeiten mit Vertretern der US-amerikanischen scritical bzw. snew musicology Kramer oder Susan McClary, mit denen er zwar den Anspruch auf eine erfahrungsgesättigte und kulturhistorisch kontextualisierte Auffassung musikalischer Bedeutung teilt, über deren essayistisch-hermeneutischen Ansatz er aber deutlich hinausgeht - vor allem

1 Neben den vor allem zu didaktischen Zwecken weiterhin höchst brauchbaren Lehrbuch-Publikationen A Guide to Musical Analysis (1987) und Analysis through Composition: Principles of the Classical Style (1996) und Büchern, die einen Schwerpunkt auf hörerrelevante Fragen musikalischer Analyse legen (Musical Analysis and the Listener, 1989; Music, Imagination, and Culture, 1990), befassen sich weitere Monographien mit einer kulturhistorischen Kontextualisierung von Musiktheorie (The Schenker Project: Culture, Race, and Music Theory in Fin-desiècle Vienna, 2007, ausgezeichnet mit dem Wallace Berry Award 2010 der Society of Music Theory) und breit angelegten fachmethodischen und musikhistorischen Themen (u.a. Rethinking Music, hg. gemeinsam mit Mark Everist, 1999, und The Cambridge History of Twentieth-Century Music, hg. gemeinsam mit Anthony Pople, 2004). durch seine enzyklopädische Sicht auf das Fach, die kulturhistorische, empirische und strukturanalytische Perspektiven in eine zugespitzte Argumentation (mitunter durch sbritischen Humor، gewürzt) einfließen lässt. Dabei gelingt es Cook, Theorie und Analyse einerseits einer rigorosen Methodenkritik zu unterziehen, sie aber gleichzeitig gegenüber den analyseskeptischen Methoden eines positivistisch gefassten Empirismus und eines kultur- und sozialhistorischen Relativismus als Erkenntnismethoden eigenen Rechts zu verteidigen.

Das Ende 2013 erschienene voluminöse Buch Beyond the Score: Music as Performance (458 S.) knüpft insofern an solche reformatorischen Bestrebungen an, als es Cook hier um nichts weniger geht als sto change the musical object from the perspective of musicology« (1). Gemeint ist damit ein mit Haut und Haaren ernst genommener, zugleich aber vielfach selbstreflexiv hinterfragter performative turn in der Musikforschung: ${ }^{2}$ Cook fordert, 'Musikı und 'performance ‘ nicht länger als zwei getrennte bzw. sich lediglich ergänzende Bereiche zu verstehen, sondern im Sinne von »music as performance» $(1)^{3}$ sich vom Paradigma des »music as writing", des textzentrierten musikalischen Ideenkunstwerks zu befreien, das Cook als »Plato's Curse» (Kap. 1) apostrophiert und in der philologisch geprägten Gründungs- und Fachgeschichte der Musikwissenschaft begründet sieht. Gewiss ist vieles von dem, was Cook in diesem Buch fordert, zwischenzeitlich weithin gängige Praxis: Rezeptionsästhetisch und aufführungspraktisch akzentuierte Forschungen haben deutlich an Gewicht und Relevanz gewonnen, nicht zuletzt auch im Zusammenhang mit der Idee eines spractice-based research ${ }^{4}{ }^{4}$

2 Vgl. Jost 2013.

3 Hervorhebung original.

4 Vgl. Doğantan-Dack 2015. 
Ihrem universellen Anspruch würde Cooks These denn auch nicht gerecht, wenn sie allzu einseitig verfolgt würde, und so übt der Autor kaum weniger explizite Kritik an Versuchen, die musikalische Schriftform zum bloßen Anlass für das Musizieren im Sinne des "musicking " ${ }^{5}$ herabzustufen (20). Und bei aller Relevanz empirischer Methodik, die gerade in der Performanceforschung viel Tradition hat, ja sich ausgehend von Carl Seashores sobjective performance analysis in den 1930er Jahren bis hin zu modernen computergestützten Methoden des sMusic Information Retrievalk (MIR) wesentlich innerhalb dieses Bereichs herausgebildet und differenziert hat, macht Cook stets klar, dass sein Erkenntnisinteresse vorwiegend ein historisches und hermeneutisches ist: $\mathrm{Er}$ möchte verstehen, in welcher Weise musikalische Bedeutung im Moment bzw. im Verlauf sowie nicht zuletzt in der historischen Aus- und Nachwirkung einer 'performances bzw. eines sperformance styles entsteht - sperformances wird also verstanden im Sinne einer »real-time activity through which meanings emerge that are not already deposited in the score« (23). Dass solche Bedeutungen nicht konstant sind, sondern starken historischen und geographischen Veränderungen, Schwankungen, mitunter aber auch paradoxen Kontinuitäten unterliegen, ist zugleich Grundvoraussetzung und fortgesetzt neu belegtes Grundthema des Buches. Es ist damit ein Plädoyer für die musikhistorische Relevanz von Interpreten und Interpretationsstilen und deren Einflussreichtum auf die Musikgeschichte, und Cook versucht so nicht zuletzt eine in der Musikforschung tief verankerte »telepathic fantasy« (31) zu widerlegen, nach der - etwa in Hugo Riemanns später Theorie der $>$ Tonvorstellungen ${ }^{6}-$ die

5 Vgl. Small 1998.

6 Riemann $(1916,2)$ hielt fest, »daß [...] gar nicht die wirklich erklingende Musik[,] sondern vielmehr die in der Tonphantasie des schaffenden Künstlers vor der Aufzeichnung in Noten lebende und wieder in der Tonphantasie des Hörers neu erstehende Vorstellung der Tonverhältnisse das Alpha und das Omega der Tonkunst ist». musikalische Kommunikation unmittelbar zwischen Komponist und Hörer verlaufe, sodass Ausführenden lediglich die undankbare Rolle zufällt, bestenfalls - bis hin zur Selbstaufgabe - dem Komponistenwillen zu dienen, ihn schlimmstenfalls aber zu verzerren und unkenntlich zu machen.

\section{,Performance Studies}

und Interpretationsforschung

Beyond the Score basiert auf nahezu 15 Jahre lang kontinuierlich betriebenen Forschungen zur Analyse und Geschichte der musikalischen Aufführung, Interpretation und sperformance und fasst so auch eine breite Anzahl von Einzelpublikationen in einen größeren Kontext zusammen, geht dabei aber deutlich über eine Aufsatzsammlung hinaus, indem bisweilen vielleicht etwas zu wortreich - konsequent Bezüge zwischen den zwölf systematisch gegliederten Kapiteln herausgearbeitet werden. Cooks Forschungen wurden ab dem Jahr 2004 in zwei großen, durch den britischen Arts and Humanities Research Council (AHRC) geförderten Forschungsprojekten gebündelt: CHARM (AHRC Research Centre for the History and Analysis of Recorded Music) an der Royal Holloway, University of London (2004-2009; http://www.charm.kcl.ac.uk) und CMPCP (AHRC Research Centre for Musical Performance as Creative Practice) an der University of Cambridge (2009-2014; http:// www.cmpcp.ac.uk). In beiden Projekten war Cook Teil eines Teams von Forschern, wobei insbesondere Eric Clarke, Daniel LeechWilkinson und John Rink gemeinsam mit Cook seit den 1990er Jahren wesentlich zur Etablierung dieser britischen sperformance studies` beigetragen haben. ${ }^{7}$ Der Kern von Cooks Methodik - die Verbindung von breiter musik- und ideengeschichtlicher Kontextualisierung, einer Herausarbeitung strukturanalytischer Details und empirisch-quantitativer Untersuchungen von Tonaufnahmen - lässt

7 Vgl. u.a. Clarke 1988, 1995; Cook 1987, 1995, 2001; Leech-Wilkinson 2001, 2009; Rink 1995, 2002. 
sich nicht zuletzt auf wechselseitige Impulse innerhalb dieses multidisziplinären Forscherteams zurückführen, ist aber schon in frühen Texten angelegt, insbesondere im Gedanken einer sperformative analysisı, die im Band Rethinking Music reziprok zu »analysing performance gedacht wurde. ${ }^{8}$ Eine nicht unerhebliche Konsequenz dieses sperformativen Analyse- und Theoriebegriffs liegt tatsächlich darin, jenem Phänomen, das analytisch, hörend oder lesend als 'Musikı bezeichnet werden kann, den Status eines klar umrissenen und gefügten (Forschungs-)>Objekts` zu nehmen, wie es die sklassische textorientierte Strukturanalyse oder auch die ontologisch orientierte Musikphilosophie voraussetzen (12f.). Anders als im Neosubjektivismus der snew musicology tegration der ästhetischen Erfahrung ein umfassend rationalisiertes Methodengerüst zugrunde, in dem die komplexe intermodale Natur musikalischer Wahrnehmung zwischen Schreiben, Lesen, Hören, Sehen, Denken, Emotion, Gesellschaft und Geschichte zu ihrem Recht kommt.

Bedauerlich ist bei all dieser bemerkenswerten multidisziplinären Ausrichtung zweifellos, dass auch Cooks Buch, wie die meisten Studien des britischen Forschungsteams, kaum die breiten und grundlegenden ,Vorarbeiten auf diesem Gebiet berücksichtigt, die in der deutschsprachigen Musikwissenschaft ausgehend von Hermann Danusers Pionierstudie Musikalische Interpretation im Neuen Handbuch für Musikwissenschaft ${ }^{9}$ geleistet wurden, besonders da einige dieser Studien durchaus bereits den von Cook verfolgten synthetischen Ansatz aus historiographischer und empirischer Methodik angesprochen oder entwickelt haben. Vor allem gilt dies für Hermann Gottschewski, der bereits im Titel seiner Dissertation Die Interpretation als Kunstwerk ${ }^{10}$ den von Cook vehement einge-

8 Vgl. Cook 1999, 248.

9 Danuser 1992. Vgl. daneben auch Danuser 1996, Danuser 1997, Hinrichsen 1999 sowie Stenzl 2012.

10 Gottschewski 1996. forderten künstlerischen `Eigenwert des Interpretationsvorgangs als Gegensatz zu einem rein textbasierten Werkbegriff ins Zentrum stellte. Auch in Hinblick auf Fragestellungen der Interpretationsgeschichte lassen sich unschwer Konvergenzen der beiden Forschungstraditionen beobachten, etwa in der von Cook wie auch von Hans-Joachim Hinrichsen ${ }^{11}$ vertretenen Position, dass eine Interpretationsgeschichte - verstanden als ein Narrativ von aufeinanderfolgenden Interpretationsstilen kaum in stringenter Weise konstruiert werden kann, sondern vielmehr >rhetorisch-freie und textzentriert sstrenge Aspekte der musikalischen Interpretation in vielen Epochen in unterschiedlicher Weise ineinandergreifen (129-133, 219-223) - sofern sie sich vor dem Beginn der Tonaufzeichnung überhaupt aus den vorhandenen Quellen, wie etwa den instruktiven gaben, extrapolieren lassen. (Ob eine breit angelegte historiographische Darstellung, wie sie in der 932-seitigen Cambridge History of Musical Performance ${ }^{12}$ versucht wird, an diesem Befund etwas ändert, muss hier offen bleiben - viel mehr als eine eher lose zusammenhängende Folge von in sich gewiss teilweise wertvollen Einzeldarstellungen scheint dieser Band jedenfalls kaum zu bieten.)

Auf der anderen Seite ist es evident, dass der Begriff sperformances, wie ihn Cook versteht, stark von Impulsen der Theater- und Medienwissenschaften ausgeht und einen umfassenderen Anspruch verfolgt als der Begriff der Interpretation - und es somit gute Gründe gibt, warum die englische Formulierung interpretation sich im Buch nur recht selten findet: Der Begriff sperformances markiert für Cook - deutlicher als jener der ,Interpretation < - eine dezidierte Gegenposition zum Verständnis musikalischer Aufführung als 'Reproduktion<, das Cook mit einer von der Theaterwissenschaftlerin Susan Melrose ${ }^{13}$ entlehnten Phrase (37) als "page-to-stage approach « (Kap. 2, 33-55) bezeichnet und das

11 Vgl. Hinrichsen 2011, $36 \mathrm{f}$.

12 Lawson/Stowell 2012.

13 Melrose 1994, 215. 
zahlreiche neusachliche und textorientierte Interpretationsschulen, insbesondere seit der ersten Hälfte des 20. Jahrhunderts prägte. Dieses Verständnis von IInterpretation ‘ als textorientierter Form der Aufführung kehrt also die bekannte Polarisierung zwischen sexécution winskys Poétique musicale gleichsam um: IInterpretation ‘ war von Strawinsky in erster Linie mit »nutzlose[n] Nuancen" und einer "Ungenauigkeit des Tempos" assoziiert worden, die dem "Geist eines Werkes « und dem Erfordernis »wirklich und aufrichtig der Musik [zu] dienen $\ll^{14}$ zuwiderlaufen. In dezidiertem Gegensatz zu einem solchen Ideal rigoroser Texttreue fasst Cook Partituren als »social scripts" auf, deren Bedeutung im Sinne einer semiotischen Übertragung erst im Moment der sperformance $<$ konkretisiert wird (Kap. 8, 249-287; vgl. 1). Dennoch wird deutlich, dass bei aller zugespitzter Verve der Argumentation der Begriff sperformance bei Cook so breit angelegt ist, dass er durchaus an etablierte Tendenzen der Aufführungspraxis- und Interpretationsforschung anschlussfähig ist..$^{15} \mathrm{Da}$ mit zeigt sich auch, dass Cooks Buch über die Polemik in Carolyn Abbates bekanntem Essay Music - Drastic or Gnostic ${ }^{16}$ hinausweist. Cooks Ansatz hebt sich von Abbate, die eine stärkere musikologische Berücksichtigung von 'Live`-Aufführungen fordert, nicht zuletzt dadurch ab, dass er den sPerformance`-Begriff sowohl auf Ton- und Videoaufnahmen als auch auf Live-Ereignisse bezieht und damit die kategoriale Trennung zwischen slive scanned music (Richard Taruksin) grundsätzlich in Frage stellt (Kap. 10 und 11; Cook schließt hier im Grunde an Taruskin an, der schon 1995 formuliert hatte: "performances, even canned performances, are not things but acts $\left.\ll^{17}\right)$. Abbates letztlich einseitige Position, die musikalische Bedeutung monokausal auf »the irreversible experience of playing, sing-

14 Strawinsky 1983, 247.

15 Vgl. Danuser 2014, 546.

16 Abbate 2004.

17 Taruskin 1995, 24. ing, or listening « $^{18}$ zurückführt, wird von Cook damit in einen breiteren kultur- und medienhistorischen Kontext gestellt.

\section{Gliederung und Thematiken}

Die sich in zahllose Stränge verästelnde Fülle seines Materials hält Cook durch eine konzise und übersichtliche Gliederung des Buches in zwei große Abschnitte und insgesamt sechs Kapitelpaare zusammen: Die ersten drei Paare (Kap. 1-6) behandeln nach zwei kultur-, musik- und fachgeschichtlich orientierten Einleitungskapiteln (Kap. 1: »Plato's Curse«; Kap. 2: "Page and Stage«) die Frage der sperformance vor allem aus der Sicht einer historisch kontextualisierten Analyse von Tonaufnahmen, wobei vorwiegend Klaviermusik des 18. und 19. Jahrhunderts im Mittelpunkt steht (Schubert, Mozart, Chopin). Dabei werden zunächst Widersprüche und Zusammenhänge zwischen analytisch-theoretischen Systemen und Erkenntnissen und einer als srhetorisch bezeichneten rubato-orientierten Aufführungspraxis in den Mittelpunkt gerückt, wobei insbesondere Heinrich Schenkers Theoriemodelle (Kap. 3: »What the Theorist Heard») und die stopic theory< sowie die historisch informierte Aufführungspraxis (Kap. 4: „Beyond Structure «) als Diskussionsgrundlage dienen. Die Kapitel 5 (»Close and Distant Listening«) und 6 (»Objective Expression«) fassen dann Ergebnisse des am Corpus der Einspielungen von Frédéric Chopins Mazurkas orientierten "Mazurka-Project" des CHARM-Forscherteams zusammen (http://mazurka.org.uk), wobei auch hier die Spannung (und teils Konvergenz) zwischen sstrukturalistischen und shetorischen Interpretationsstilen in breite kultur- und mediengeschichtliche Kontexte eingebettet wird. Statistische Methoden, teilweise durch computergestützte Verfahren optimiert und veranschaulicht, werden hierbei mit differenzierten musikhistorischen und strukturanalytischen Befunden quergelesen.

Im zweiten Teil des Buches dann wird die Perspektive deutlich breiter auf kulturhistori-

18 Abbate 2004, 505. 
sche und soziologische Kontexte der sperformancer gelenkt und im Zusammenhang damit auch ein heterogeneres Repertoire behandelt, von Jimi Hendrix, Jazz-Improvisation und snew complexity، bis zu Arcangelo Corelli und dem Streichquartett des klassischen Stils, wobei Chopins Mazurkas einen mehrfach wiederkehrenden $>$ Refrain bilden. In den Kapiteln 7 (»Playing Somethin'«) und 8 (»Social Scripts») ist der Gedanke des Musizierens als sozialer Aktivität entwickelt, wobei den vielfältigen Formen der Interaktion zwischen Musikern, etwa in Kammermusik oder in Improvisationsensembles, besonderes Gewicht zukommt und ethnographische Methoden vorgestellt werden, die versuchen, das hierbei zur Anwendung kommende sintuitive Wissen Interpreten zu dokumentieren und zu analysieren - eine Dimension, die längst zu einem breiten eigenständigen Forschungsfeld geworden ist und rein empirische Methoden ${ }^{19}$ ebenso umfasst wie ethnographisch-deskriptive. ${ }^{20}$ Cook pointiert hierbei seine Kritik am textorientierten Werkbegriff in der These vom »work as performance (237-248). Das musikalische Werk erscheint als irreduzible soziale Konstruktion (239): Anhand der unterschiedlichen Editionen von Corellis Sonaten op. 5 und anderer Beispiele wie Jazz-Improvisation versucht Cook die Position zu dekonstruieren, ein Werk zeichne sich durch eine bestimmte Konstellation unverwechselbarer struktureller Eigenschaften aus, die durch den Interpreten lediglich svermittelt oder sübersetzt ‘ werden müssten. Cooks Plädoyer für das »work as performance " versteht sperformances im Gegensatz dazu als semiotische Aktivität, "which does not set out to reproduce the structure literally or exhaustively. Instead, it is based on a system of abbreviations and cues, and this contributes to the irreducibly historical dimension of performance - and indeed of listening - that we call style. [...] performers do not reproduce structure: rather they reference or signify it." (245) Dies hat nicht zuletzt zur Folge, dass die Bedeutung der makro-

19 Vgl. z. B. Goebl/Palmer 2009.

20 Vgl. z. B. Davidson/Goode 2002. formalen Anlage von Werken, die in der strukturalistischen Fundierung des Werkbegriffs im Mittelpunkt steht, in Cooks sperformativems Werkbegriff einer Hinwendung zu flüchtigen Momenten der Emergenz und Referenz weicht.

Darauf stellen die Kapitel 9 (»The Signifying Body») und 10 (»Everything Counts«) die Dimension des Körpers in den Vordergrund, wobei die "construction of meaning through embodied practice« (6) an so unterschiedlichen Beispielen wie Jimi Hendrix' Foxy Lady und Analysen von Videoaufnahmen der Chopin-Mazurka op. 63/3 verhandelt wird. Ziel ist es dabei, die durch dieses sembodiment entstehenden Bedeutungsebenen mit jenen querzulesen, die quantitativ orientierte Tonträgeranalysen auf Corpusebene zu Tage fördern, wie sie in Kapitel 6 vorgestellt wurden. Kapitel 11 (»The Ghost in the Machine schließlich unternimmt einen breit angelegten medienhistorischen Ausblick auf die Entwicklung von Musikaufnahmen seit den Anfängen mit Phonograph und Grammophon, wobei als zentrale These hier nochmals der Gegensatz von sperformances und reproductions auf den Punkt gebracht wird: 'Performances‘ sind für Cook mehr als Reproduktionen von Werken und umgekehrt sind Musikaufnahmen mehr (oder jedenfalls etwas anderes) als Reproduktionen von sperformances،. Cook problematisiert Bestrebungen, durch Aufnahmen Aufführungen zu suggerieren (und damit potenziell zu ersetzen), während zugleich versucht werde, durch die hohe Perfektion von Live-Konzerten medialisierte Hörerfahrungen zu imitieren - ein Teufelskreis, der laut Cook mit zur globalen Marginalisierung von Kunstmusik (im Buch als WAM = , Western Art Music abgekürzt) beitrage: Die Möglichkeiten von sperformances, Bedeutungen zu entfalten, die über den Gedanken der sreproduction hinausweisen, wie sie im Schlusskapitel zusammengefasst sind (Kap. 12, „Beyond Reproduction«), würden so vergeben. Andererseits zwingt dieser Befund dazu, auch den gesamten Produktionsprozess von musikalischen Aufnahmen als Teil jener sperformance s anzusehen, die durch diese Aufnahmen suggeriert wird, also auch Aufnahmetechnik, Mastering 
etc. verstärkt in die Analyse mit einzubeziehen, was mit der Forderung einer umfassenden Quellenkritik im Bereich der Erforschung von Tonträgern einhergeht (Kap. 5, 139-143).

\section{,Performance Analysis`}

Von besonderem Interesse für die Musiktheorie ist zweifellos der erste Teil von Beyond the Score und dabei insbesondere die Hauptkapitel 3 bis 6, zum einen da sie sich mit gängigen Theorie- und Analysesystemen auseinandersetzen und mittels der sperformance analysis` deren Anspruch auf Allgemeingültigkeit dekonstruieren, zum anderen weil hier jenes Kernrepertoire der europäischen (Klavier-)Musik im Zentrum steht, das Grundlage aller einflussreichen neueren Musik- bzw. Tonalitätstheorien bildet. Grundlegend für Cooks Verfahren ist die Kritik am universellen Anspruch von Analyse und Theorie, insbesondere dort, wo diese sich anmaßen, im Sinne des »analysis-to-performance approach « (49f.) auch verbindliche Richtlinien für die musikalische Ausführung vorzugeben. Cook will demgegenüber zeigen, dass die ranalytisches oder istrukturalistische` Form der Ausführung nur ein Interpretationsstil unter vielen ist, der erst nach dem Zweiten Weltkrieg im Zuge der Verbreitung entsprechender Ideale in der Musikerausbildung global Akzeptanz fand (56). Eine umfangreiche Fallstudie, die Cook bereits 2008 auf dem Grazer Kongress der Gesellschaft für Musiktheorie vorstellte ${ }^{21}$ und die den Kern des 3. Kapitels von Beyond the Score bildet, betrifft Heinrich Schenkers Stimmführungsanalyse und ihren Bezug zur Aufführungspraxis. Dabei stellt sich zunächst die Schwierigkeit, eine konzise Aufführungslehre aus Schenkers Schriften zu rekonstruieren, wozu neben der Fragment gebliebenen Schrift Die Kunst der Vortrags, die 2000 in einer englischsprachigen Edition erschien ${ }^{22}$, vor allem eine Reihe von Interpretationshinweisen dienen können, die Schenker in viele seiner Veröffentlichungen integrierte, so auch

21 Cook 2010.

22 Schenker 2000. in seine in Der Tonwille publizierte Analyse von Franz Schuberts Impromptu Ges-Dur op. 90/3. ${ }^{23}$ Bereits in Kapitel 2 differenziert Cook im Detail, dass weder Schenkers eigene Ausführungen zur Aufführung noch jene der US-amerikanischen Nachkriegs- und Gegenwartstheorien, die versuch(t)en, Schenkers Theoriesystem für die Aufführungspraxis fruchtbar zu machen, auf ein naives Modell der swörtlichen analyse in Tempo- und Dynamikvorschriften reduziert werden können (34-40). Im Gegenteil gibt es sowohl von Schenker selbst als auch von 'Schenkerianischen Theoretikern hinreichend Belege für die Haltung, dass das `Herausbringen oder sProjizieren Struktur bei der Aufführung nur eine mögliche Option für die Interpreten sei und keinesfalls übertrieben eingesetzt werden dürfe ${ }^{24}(44 \mathrm{f}$.). Dem steht nun aber eine stark autoritative Tendenz in Schenkers Theoriefragment zur Aufführungslehre gegenüber, in der davon ausgegangen wird, dass jedes Werk nur eine

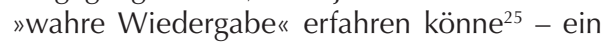
Topos, der sich in einer vergleichbaren, wenn auch offener formulierten Form auch bei Rudolf Kolisch und Theodor W. Adorno findet. ${ }^{26}$ Indem Cook nun eine detaillierte Analyse der Einspielung von Schuberts Impromptu auf einer Welte-Mignon-Klavierrolle (1905) durch Eugen d'Albert - einen von Schenker erklärtermaßen favorisierten Pianisten - mit Schenkers Stimmführungsanalyse einerseits und Schenkers Interpretationsanweisungen andererseits konfrontiert und diesen Diskurs dann mit universalistischen Theorien der Tempo- und Phrasengestaltung ${ }^{27}$ sowie mit älteren und neueren Aufnahmen des Schubert-Werkes (Edwin Fischer, Artur Schnabel, Murray Perahia u.a.) kontextualisiert, stellt er grundlegende Paradoxien im Verhältnis von Analyse und Aufführung heraus: Nicht nur

23 Schenker 1924.

24 Schenker 1956, 34f.; Rothstein 1995, 218.

25 Schenker 2000, 77 (Übers. des Verf.).

26 Kolisch 1983, 13f.; Adorno 2001, 120.

27 Vgl. Cone 1968; Todd 1992. 
gibt es unauflösliche Widersprüche zwischen der strukturalistischen (`modernist`) Analysemethode Schenkers und seinen ganz in der srhetorischen` (spremodernist`) Tradition stehenden Vorstellungen eines mobilen Tempos und nuancenreichen Vortrags; auch die von strukturalistisch orientierten Interpretationslehren und musikpsychologischen Studien propagierte `Natürlichkeit einer am Notentext orientierten Strategie des sphrase-final lengthening bzw. sphrase arching - vor dem Hintergrund der Gravitationsmetapher basierend auf der Analogie zwischen einer bogenförmigen Tempo- und Dynamikgestaltung von musikalischen Phrasen mit dem Wurf eines Steins oder Balls ${ }^{28}$ - erweist sich als Konstrukt, das einer historisch-kritischen Untersuchung der Interpretationsstile nicht standhält. Zwar zeigt der Vergleich von insgesamt acht Einspielungen des Schubert-Impromptus aus dem Zeitraum 1905-1997 eine vage Tendenz zur Reduzierung von Temposchwankungen und zu einem langsameren Grundtempo sowie eine nach 1945 wachsende Bereitschaft, die IIdeologie des sphrase arching ‘ umzusetzen, allerdings bezeugen die Aufnahmen zugleich die kontinuierliche Relevanz von irregulären Hervorhebungen, die gerade nicht durch strukturelle Analyse erklärbar sind und die Cook mit Verweis auf die Beschreibung von raccents pathétiques` im Traktat Mathis Lussys ${ }^{29}$ besonders herausstellt (69). Vor dem Hintergrund seiner Exkurse in die Bereiche der energetischen Musiktheorie sowie der Affekt- und Emotionsforschung will Cook damit auch die Variabilität von emotionalen Gehalten zeigen, wie es dem emergenten Strukturbegriff entspricht: »emotional meaning is not inherent in the musical work but arises from the contexts of its interpretation, understanding that word in the broadest sense." (79) Cook zieht aus der Diagnose eines unbewältigten Konflikts zwischen einem praxisbezogenen Ideal freier, shetorischer Interpretationsstilistik und der

28 Bereits bei Christiani 1885, 160, dann vor allem bei Cone 1968, 26-31, und schließlich als kognitivistisches Modell bei Todd 1992.

29 Vgl. Lussy 1874, 11.
Verabsolutierung eines textzentrierten Werkverständnisses die Folgerung, dass sowohl Schenkers als auch Adornos und Kolischs geplante Monographien zur musikalischen Interpretation letztlich daran scheiterten, dass sie das Paradigma der Reproduktion nicht aufgeben wollten, zugleich aber eine starke Beziehung zur Interpretationspraxis der Jahrzehnte um 1900 pflegten, die einem solchen Paradigma drastisch widersprochen habe (89). Eine gewisse Einseitigkeit liegt wohl in Cooks Wertung von Adornos ohnehin nur stark fragmentarisch überlieferten und heterogenen Skizzen zur Aufführungstheorie, galt für Adorno doch keineswegs naive Texttreue als Maßstab einer "wahre[n] Reproduktion«. Diese sei eben »nicht einfach die Realisierung des Befundes der Analyse«: »Das ergäbe einen unerträglichen Rationalismus und setzte tendenziell die Musikwissenschaft als Instanz der musikalischen Darstellung ein «. ${ }^{30}$

Die Kapitel 4 bis 6 knüpfen an diese Diskussion mit äußert materialreichen Darstellungen an. Dabei zeigt Kapitel 4 anhand zweier Sätze aus Mozarts Klaviersonaten (KV 332, 1. Satz, und KV 331, 3. Satz Alla Turca) erneut eingehend die kontinuierliche Relevanz rhetorischer Interpretationsstrategien vor dem Hintergrund der historisch informierten Aufführungspraxis. Insbesondere die Orientierung an musikalischen Topoi, durch die eine $>d r a-$ matischer Lesart der klassischen Phrasenstrukturen motiviert ist, führt etwa bei Einspielungen von Hammerklavier-Solisten wie Malcolm Bilson oder Bart van Oort zu einer teilweise eklatanten 'Mobilität des Tempos, das aber durchaus in den Dienst istrukturalistischer Lesarten gestellt wird - in Gegensatz zu den mitunter verblüffenden saccents pathétiquess der Generation um 1900, die hier u. a. durch Aufnahmen Carl Reineckes vertreten ist. Die Coda des Alla Turca setzt van Oort durch eine drastische Verlangsamung als formal eigenständigen Formteil ab und zelebriert dabei Phrasenverschränkungen und -verkürzungen. In solchen Detailanalysen zeigt sich, wie sehr sich das istructure-to-performance paradigmı,

30 Adorno 2001, 106f. (Hervorhebung original). 
gegen das Cooks Buch so massive Kräfte mobilisiert, immer wieder als äußerst produktive Reibungsfläche erweist.

Dasselbe gilt für die in den Kapiteln 5 und 6 nun auf breiter Repertoirebasis und methodisch stark durch quantitative Verfahren erweiterte Darstellung von historischen Dimensionen der Chopin-Interpretation. Die Reibungsfläche bieten hier wiederum die >Ideologien ‘ des sphrase arching`, die in einem breiten kulturhistorischen Exkurs als Grundlage des modernen Paradoxes eines sobjektiven`, da vermeintlich snatürlichen Ausdrucks herausgestellt und in Verbindung gebracht werden mit so unterschiedlichen Manifestationen wie dem Ideal der Schlichtheit in Mode (Coco Chanel; 212) oder funktionaler Architektur (Le Corbusier; 213-216) oder den nachhaltigen - und von Adorno beklagten - Tendenzen zur Standardisierung, wie sie moderne Aufnahme- und Reproduktionstechnologien mit sich brachten (217f.). Die Vorstellung musikalischer Form als `Architektonikı wurde dabei - im Namen Strawinskys - nicht zuletzt durch Nadia Boulanger in Theorie und Praxis vertreten, wobei auch hier - vermeintlich im Zenit der neusachlichen sstrukturalistischen Aufführungspraxis - die Tondokumente eine kontinuierliche Relevanz ,rhetorischer Topoi bezeugen (221 f.).

Die als Resultat der CHARM-Forschungen vorgestellten quantitativen Erhebungen zur Aufnahmegeschichte von Chopins Mazurkas gehen von der These aus, dass solche Corpusstudien durch `distant listening`im Sinne des Literaturtheoretikers Franco Moretti einerseits gewisse Vorteile bieten, etwa das Vermeiden von tautologischen Forschungsergebnissen, in denen nur das herausgehoben wird, was die Forscher in die Aufnahmen shineinhören`. Andererseits bedürfen, wie Cook mehrfach verdeutlicht, solche Methoden stets der qualitativen Überprüfung durch ein `close listening`, sodass mikro- und makroskopische Perspektiven sich fortgesetzt wechselseitig kommentieren können. Kapitel 5 gibt dabei vorwiegend dem `close listening، den Vorzug, nicht zuletzt mit der Intention, die eminent politische und nationalistische Rezeptionsgeschichte, die gerade mit Chopins Mazurkas verbunden ist, anhand der Interpretationsgeschichte der besonders sidiomatischen Mazurka op. 33/2 (D-Dur) zu dekonstruieren. Die in Kapitel 6 vorgestellten, mit Hilfe von Cooks Mitarbeiter Craig Sepp mittels der Software Sonic Visualizer (http://www.sonicvisualiser.org) und des Scape Plot Generator (http://www.mazurka. org.uk/software/online/scape) durchgeführten Corpusstudien zu Einspielungen der Mazurkas op. 63/3 (cis-Moll; 34 Aufnahmen aus dem Zeitraum 1923-2003) und op. 17/4 (a-Moll, sieben Aufnahmen aus dem Zeitraum 19392002) versuchen dann über eine integrierte graphische Darstellung von Tempo- und Dynamikgestaltung historische Tendenzen des sphrase arching ‘ zu beschreiben. Auch wenn die graphischen Darstellungen des Scape Plot Generator gewöhnungsbedürftig und kaum intuitiv lesbar sind, so zeigt Cook doch in diesem Kapitel, wie facettenreich die sperformance analysis auch gerade auf dieser Corpusebene sein kann und wie nachhaltig sie durch das sclose listening، immer wieder neu befruchtet und vertieft werden kann (wie auch für andere Kapitel erlaubt hier die Companion website zum Buch einen detaillierten Nachvollzug anhand ausgewählter Audiound Analysedateien; http://global.oup.com/us/ companion.websites/9780199357406). Wenig verwunderlich ist, dass Cooks Grundthese schließlich auch hier bestätigt wird: Zwar nahm ein Tempo- und Dynamikgestaltung integrierendes sphrase arching، (das hier einleitend vor allem mit Hugo Riemanns einflussreicher, wenn auch bereits zu ihrer Entstehung stark umstrittener Phrasierungslehre in Verbindung gebracht wird, 178-182) nach 1945 in deutlichem Ausmaß zu und bezeugt den Einfluss eines pädagogisch institutionalisierten sstrukturalistischen ' Interpretationsstils, zugleich aber bleiben srhetorischer Abweichungen von diesem textorientierten Ideal bis in die Gegenwart kontinuierlich präsent, nicht zuletzt da viele Interpreten von der in Todds Modell vorausgesetzten Koppelung von Tempo- und Dynamikbögen nachhaltig abweichen. Die Chimäre einer 'Natürlichkeitı dieses Modells wird damit ebenso offenbar wie die 
kulturpessimistische Klage über die vermeintliche Vereinheitlichung der Interpretationsstile im Zuge der Reproduzierbarkeit und den Verlust an Nuancierung, wie sie nicht zuletzt Schenker und Adorno anstimmten, durch die Heterogenität dieser Gesamtschau eindrucksvoll widerlegt wird.

\section{Herausforderungen}

Nicholas Cooks Buch bietet reiches Material, das bei aller mitunter skriminologischen، Zuspitzung (etwa im Abschnitt »Forensics vs. Musicology", 149-157, in dem es um die sAufdeckung, von Aufnahmen geht, die unter falschem Interpretennamen veröffentlicht wurden) doch gewiss auch Längen und eine mitunter redundante Wiederkehr von Argumenten aufweist, nichtsdestotrotz aber voller Anregungen und Herausforderungen für alle musikologischen Fachgebiete steckt und insofern das selbst gesteckte Ziel sto change the musical object from the perspective of musicology« (1) ohne Zweifel ein beträchtliches Stück vorangetrieben hat. Bei alledem muss festgehalten werden, dass Cook in diesem Buch fast durchweg sperformance analysis und kaum sperformative analysis betreibt: Analyse im emphatischen Sinn ist so gut wie nicht anzutreffen, der Analysebegriff dient zumeist als eher abstrakt bleibende Negativfolie der Argumentation. Das Potenzial, auf dem Weg einer integrierten Untersuchung von Aufnahmen und Aufführungen zu neuen strukturell-analytischen Deutungen der Werke zu gelangen, wird kaum eingelöst, und wenn, dann nur auf der relativ eindimensionalen Ebene der Phrasensyntax. Viele von Cooks Argumentationen sind dabei zugleich nur vor dem Hintergrund jener sformalistischen hermeneutischen Analysetraditionen verstehbar, die auf das Engste mit dem Denken in den und durch die europäischen KunstmusikTraditionen verbunden sind und irreversibel auf diese eingewirkt haben. Durch ihre Ausklammerung tendiert Cook dazu, das Kind mit dem Bade auszuschütten (und dass sich in der Tat ein Kind in der Wanne befindet, räumt Cook in einem vergleichbaren Zusammenhang selbst ein, wenn er seinerseits Carolyn Abbate vorwirft, Musikaufnahmen vom sperformances-Begriff auszuschließen, 328). Ein anderes Desideratum, das Cooks Buch weitgehend offen lässt, ist die Frage, inwiefern (oder mit welchen Modifikationen) der Ansatz der sperformance analysis` auf vor 1750 und nach 1900 komponierte Musik anwendbar ist. Die lesenswerte ethnographische Studie zur Einstudierung eines in der Tradition der snew complexity، komponierten Klavierstücks im Abschnitt »Performing Complexity« (273-287, bereits 2005 erstmals veröffentlicht ${ }^{31}$ ) bietet aufgrund ihrer methodisch sehr spezifisch gearteten Herangehensweise dafür nur bedingt Anhaltspunkte. In Bezug auf neue Musik ist die naheliegende Vermutung, der sstrukturalistische، Interpretationsstil sei hier verabsolutiert worden und zur unhinterfragten Norm geworden, jedenfalls gewiss nicht haltbar ${ }^{32}$, und diese Erkenntnis lässt sich ohne Zweifel auch auf eine längst von einem philologischen Historismus abgekommene Aufführungspraxis von Barock- und Renaissance-Musik übertragen. Gerade auch eine sperformance entierte Forschung in diesen Bereichen wird ohne Zweifel stark von Cooks in ihrer Breite und Weitsicht einzigartiger Darstellung profitieren können.

Christian Utz

31 Clarke/Cook/Harrison/Thomas 2005.

32 Vgl. Utz 2016. 


\section{Literatur}

Abbate, Carolyn (2004), »Music - Drastic or Gnostic?", Critical Inquiry 30, 505-536.

Adorno, Theodor W. (2001), Zu einer Theorie der musikalischen Reproduktion, Frankfurt a. M.: Suhrkamp.

Christiani, Adolph (1885), The Principles of Expression in Pianoforte Playing, New York: Harper \& Brothers.

Clarke, Eric (1988), „Generative Principles in Musical Performance«, in: Generative Processes in Music: The Psychology of Performance, Improvisation, and Composition, hg. von John Sloboda, Oxford: Oxford University Press, 1-26.

- (1995), »Expression in Performance: Generativity, Perception, and Semiosis", in: The Practice of Performance, hg. von John Rink, Cambridge: Cambridge University Press, 21-54.

Clarke, Eric / Nicholas Cook / Bryn Harrison / Philip Thomas (2005), »Interpretation and Performance in Bryn Harrison's êtretemps«", Musicae Scientiae 9, 31-74.

Cone, Edward T. (1968), Musical Form and Musical Performance, New York: Norton.

Cook, Nicholas (1987), "Structure and Performance Timing in Bach's C major Prelude (WTC 1): An Empirical Study", Music Analysis 6, 257-272.

- (1995), »The Conductor and the Theorist: Furtwängler, Schenker, and the First Movement of Beethoven's Ninth Symphony", in: The Practice of Performance: Studies in Musical Interpretation, hg. von John Rink, Cambridge: Cambridge University Press, 105-125.

- (1999), »Analysing Performance and Performing Analysis«, in: Rethinking Music, hg. von Nicholas Cook und Mark Everist, Oxford/New York: Oxford University Press, 239-261.

- (2001), »Between Process and Product: Music and/as Performance«, Music Theory Online 7. http://www.mtosmt.org/ issues/mto.01.7.2/mto.01.7.2.cook.html

- (2010), „Struktur und Interpretation. Eugen d'Alberts und Heinrich Schenkers
Deutungen von Franz Schuberts Impromptu op. 90,3 im historischen Kontext", in: Musiktheorie als interdisziplinäres Fach. 8. Kongress der Gesellschaft für Musiktheorie Graz 2008 (= musik.theorien der gegenwart 4), hg. von Christian Utz, Saarbrücken: Pfau, 265-288.

Danuser, Hermann (Hg.) (1992), Musikalische Interpretation $(=$ Neues Handbuch der Musikwissenschaft 11), Laaber: Laaber.

- (1996), »Interpretation", in: Die Musik in Geschichte und Gegenwart. Allgemeine Enzyklopädie der Musik, 2. Aufl., hg. von Ludwig Finscher, Sachteil Bd. 4, Kassel u. a.: Bärenreiter u. a., 1053-1069.

- (1997), „VII. Musikalische Interpretation«, in: Im Zenit der Moderne. Die Internationalen Ferienkurse für Neue Musik Darmstadt 1946-1966, Bd. 2, hg. von Gianmario Borio und Hermann Danuser, Freiburg: Rombach, 119-187.

- (2014), »Exekution - Interpretation - Performance. Zu einem begriffsgeschichtlichen Konflikt« [2009], in: ders., Gesammelte Vorträge und Aufsätze, Bd. 1, hg. von Hans-Joachim Hinrichsen, Christian Schaper und Laure Spaltenstein, Schliengen: Argus, 530-548.

Davidson, Jane / James Goode (2002), „Social and Musical Co-ordination Between Members of a String Quartet: An Exploratory Study", Music Psychology 30, 186-201.

Doğantan-Dack, Mine (Hg.) (2015), Artistic Practice as Research in Music: Theory, Criticism, Practice, Farnham: Ashgate.

Goebl, Werner / Caroline Palmer (2009), "Synchronization of Timing and Motion Among Performing Musicians", Music Perception 26, 427-438.

Gottschewski, Hermann (1996), Die Interpretation als Kunstwerk. Musikalische Zeitgestaltung und ihre Analyse am Beispiel von Welte-Mignon-Klavieraufnahmen aus dem Jahre 1905 (= Freiburger Beiträge zur Musikwissenschaft 5), Laaber: Laaber.

Hinrichsen, Hans-Joachim (1999), Musikalische Interpretation. Hans von Bülow 
(= Beihefte zum Archiv für Musikwissenschaft 46), Stuttgart: Steiner.

- (2011), „Kann Interpretation eine Geschichte haben? Überlegungen zu einer Historik der Interpretationsforschung", in: Gemessene Interpretation. Computergestützte Aufführungsanalyse im Kreuzverhör der Disziplinen, hg. von Heinz von Loesch und Stefan Weinzierl, Schott: Mainz, 27-37.

Jost, Christofer (2013), »Der sperformative turn Desiderat und (teil)disziplinärem Paradigma", Musiktheorie 28, 291-309.

Kolisch, Rudolf (1983), Zur Theorie der Aufführung. Ein Gespräch mit Berthold Türcke (= Musik-Konzepte 29/30), München: edition text + kritik.

Lawson, Colin / Robert Stowell (Hg.) (2012), The Cambridge History of Musical Performance, Cambridge: Cambridge University Press.

Leech-Wilkinson, Daniel (2001), „Using Recordings to Study Musical Performance", in: Aural History: Essays on Recorded Sound, hg. von Andy Linehan, London: The British Library, 1-12.

- (2009), The Changing Sound of Music: Approaches to Studying Recorded $\mathrm{Mu}$ sical Performances, London: AHRC Research Centre for the History and Analysis of Recorded Music. http://www.charm. rhul.ac.uk/studies/chapters/intro.html

Lussy, Mathis (1874), Traité de l'expression musicale. Accents, nuances et mouvements dans la musique vocale et instrumentale, Paris: Heugel.

Melrose, Susan (1994), A Semiotics of the Dramatic Text, Basingstroke u.a.: Macmillan.

Riemann, Hugo (1916), »Ideen zu einer >Lehre von den Tonvorstellungen«", Jahrbuch der Musikbibliothek Peters 21/22, 1-26.

Rink, John (1995), »Playing in Time: Rhythm, Metre and Tempo in Brahms's ,Fantasien op. 116", in: The Practice of Performance: Studies in Musical Interpretation, hg. von John Rink, Cambridge: Cambridge University Press, 254-282.

- (2002), "Analysis and (or?) Performance", in: Musical Performance. A Guide to Un- derstanding, hg. von John Rink, Cambridge: Cambridge University Press, 35-58.

Rothstein, William (1995), „Analysis and the Act of Performance", in: The Practice of Performance: Studies in Musical Interpretation, hg. von John Rink, Cambridge: Cambridge University Press, 217-240.

Schenker, Heinrich (1924), "Schubert: Quatre Impromptus, op. 90, Nr. 3《, in: Der Tonwille. Flugblätter zum Zeugnis unwandelbarer Gesetze der Tonkunst 4/4 [= H. 10], 14-21.

- (1956), Der freie Satz (= Neue musikalische Theorien und Phantasien 3) [1935], 2. bearbeitete Auflage, hg. von Oswald Jonas, Wien: Universal Edition.

- (2000), The Art of Performance [1911], hg. von Heribert Esser, New York: Oxford University Press.

Small, Christopher (1998), Musicking: The Meanings of Performing and Listening, Hanover $(\mathrm{NH})$ : Wesleyan University Press.

Stenzl, Jürg (2012), Auf der Suche nach Geschichte(n) der musikalischen Interpretation, Würzburg: Königshausen \& Neumann.

Strawinsky, Igor (1983), »Musikalische Poetik« [1942], in: ders., Schriften und Gespräche I, Mainz u. a.: Schott, 173-256.

Taruskin, Richard (1995), »Introduction: Last Thoughts First", in: ders., Text and Act. Essays on Music and Performance, New York/Oxford: Oxford University Press, $3-47$.

Todd, Neil (1992), »The Dynamics of Dynamics. A Model of Musical Expression «, Journal of the Acoustical Society of America 91, 3540-3550.

Utz, Christian (2016), »Time-Space Experience in Works for Solo Cello by Lachenmann, Xenakis, and Ferneyhough. A Performance-Sensitive Approach to Morphosyntactic Musical Analysis", Music Analysis Early View (2016), doi: 10.1111/ musa.12076. http://onlinelibrary.wiley. com/doi/10.1111/musa.12076/full 